\title{
ABC4MAS: Assembling business collaborations for MAS
}

\author{
T. Penya-Alba*, M. Pujol-Gonzalez*, M. Esteva*, B.Rosell*, J. Cerquides*, J.A. Rodriguez *, C. Sierra*, C. Carrascosa ${ }^{\dagger}$, \\ V. Julián ${ }^{\dagger}$, M. Rebollo ${ }^{\dagger}$, M. Rodrigo ${ }^{\dagger}$ and M. Vasirani ${ }^{\ddagger}$ \\ *IIIA-CSIC \\ ${ }^{\dagger}$ Universitat Politècnica de Valencia \\ ${ }^{\ddagger}$ Universidad Rey Juan Carlos
}

\begin{abstract}
ABC4MAS provides an environment to support the rapid assembly of agent-oriented business collaborations. ABC4MAS allows: (i) to set up a collaboration environment (CE) as a virtual organisation; (ii) to reach agreements within the $\mathrm{CE}$ to form short-term business collaborations; (iii) to enact business collaborations; and (iv) to track the performance of agents within business collaborations to build their trust and reputation within the $\mathrm{CE}$.
\end{abstract}

Keywords-virtual organizations, agent platforms, combinatorial auction, supply chain formation, multiagent system

\section{INTRODUCTION}

Globalization and technological innovation are driving the creation of the extended enterprise - the dynamic network of interconnected organisations, from suppliers' suppliers to customers' customers, which work collaboratively to bring value to the marketplace. Hence, today's companies are in need for support to swiftly create business collaborations that allow them to readily respond to changing market needs. The capability of forming and sustaining collaborations has become central for companies.

The ABC4MAS demonstrator provides an environment to support the rapid assembly of business collaborations whose players employ agent technology. Concretely, the demonstrator allows: (i) to set up a collaboration environment (CE) as a virtual organisation; (ii) to reach agreements within the $\mathrm{CE}$ to form short-term business collaborations; (iii) to enact business collaborations; and (iv) to track agents' performances to build their trust and reputation.

\section{BASE TECHNOLOGY}

ABC4MAS builds upon and extends several agentoriented technologies. Next, we briefly describe the functionality of each one of the ABC4MAS building blocks.

THOMAS. The THOMAS framework [1] allows to dynamically create, manage and destroy Virtual Organisations (VOs) that seamlessly integrate agents, norms and services. THOMAS can manage organisational structures along with their norms. Moreover, it controls the visibility and access to the services within a VO. All the functionalities are offered as semantic web services. On the one hand, the Service Facilitator (SF) handles the discovery of and access to services by agents and organisations. On the other hand, the Organisation Management System (OMS) is in charge of VO life-cycle management, including specification and administration of both the structural components of the organisation (roles, units and norms) and its execution components (participant agents and roles they play).

EIDE. The Electronic Institutions Development Environment (EIDE) [2] is a set of software tools that support all the stages in the engineering of an electronic institution (EI). The ISLANDER tool allows designers to graphically define a formal specification of the rules of an electronic institution according to a formal language. The SIMDEI tool allows to run EI simulations with different agent populations. Thus, SIMDEI enables EI designers to analyze simulation results and decide whether the institutional rules yield the expected behavior or should be tweaked. EIDE also includes the aBUILDER tool that supports the graphical specification of agent behaviors starting from an institution specification created with ISLANDER. Finally, EIDE includes the AMELI tool, an execution environment for EIs. AMELI keeps validating agents' actions using the institutional rules along with the state of the institution.

MMUCATS. MMUCATS is a test suite for mixed multi-unit combinatorial auctions (MMUCAs) [3]. MMUCAs offer a high potential to solve supply chain formation problems, and thus be employed for the automated assembly of supply chains of agents. MMUCATS includes optimal solvers that allow to solve a MMUCA winner determination problem. MMUCATS allows to automatically generate the workflow for the optimal supply chain structure produced by a winner determination algorithm as an ISLANDER specification. Therefore, the EI generated by the MMUCATS solvers can be fed into AMELI to enact a supply chain as an EI.

SRM. The Supplier Relationship Management (SRM) tool [4] allows to track how well agents perform their contracts. It is based on a measure of trust based on a knowledge base containing past experiences obtained after agents deliver their contracts. Each experience is composed of a commitment (corresponding to a contract) along with the observation of the execution of the commitment. This allows to deal with multiple requirements, including: (1) the importance of each contract's attribute; (2) how different is what you got compared with what you asked for and, of course, (3) which are your preferences.

\section{ARChitecture OVERVIEW}

ABC4MAS integrates the base technologies presented in section II. With this aim, all the building blocks of the demonstrator have been developed as OSGI services. OSGI provides standardized primitives that allow applications to be 
constructed dynamically from small, reusable and collaborative components. Next, we describe how the ABC4MAS services orchestrate to launch, run, and monitor business collaborations.

First of all, the demonstrator starts out from the specification of a collaboration environment (CE). The specification includes all participants that may take part in the process of providing goods/services, including both external and internal resources/agents. Moreover, the specification includes the roles each participant can play. A CE definition is fed as a new THOMAS service, which in turn will create a virtual organisation representing the whole $\mathrm{CE}$, as shown in Figure 1(a).

Thereafter, the CE can start receiving requests for performing services. After a request is received, the agents in the virtual organisation compete for being part of the collaboration that allows to deliver the requested service. With this aim, an auctioneer agent within the CE initiates a MMUCA auction offering it as a new THOMAS service. After receiving bids for supply chain operations from the agents in the CE, the auctioneer calls the MMUCATS service to solve the winner determination problem (WDP) and hence assess the resulting collaboration as a supply chain structure. Once an auctioneer closes its auction, it calls THOMAS to start out a new collaboration (as a virtual organisation) whose participants and roles correspond to those in the optimal supply chain assessed by MMUCATS. To clarify, the whole process involved in forming a short-term collaboration to fulfill a service request is depicted in Figure 1(b).

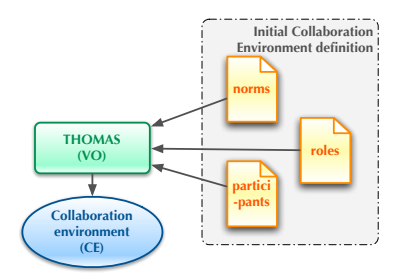

(a) Collaboration environment creation.

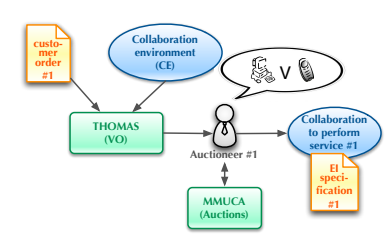

(b) Collaboration formation.
Figure 1. ABC4MAS main processes

Once a new collaboration has been assessed, it is time to enact it to allow the agents in the collaboration perform the operations they committed to. With this aim, the auctioneer launches an AMELI service. AMELI then tracks and validates every action of the agents in the collaboration to ensure that each agent timely performs the operations he committed to. Additionally, AMELI generates events corresponding to all the transactions taking place between agents. Events are stored in a central event database shared by all virtual organisations spawned from the CE. When AMELI detects that the collaboration has finished (the service causing the creation of the collaboration has been performed), AMELI terminates the Electronic Institution running the collaboration process.
During the performance of a service, the SRM service interacts with the central event database to feed its trust and reputation model. Hence, the agents in the $\mathrm{CE}$ can employ the SRM services to evaluate agents' performances. Moreover, both the trust and reputation information are made available to the agents in the CE. For instance, an auctioneer might use this information to filter out bids from agents that underperformed when taking part in past collaborations. As a result, subsequent collaborations may turn out to be more resilient to underperforming agents.

Finally, Figure 2 shows a diagram of the complete workflow, from the creation of the $\mathrm{CE}$ as a virtual organisation to the performance of multiple services in response to service requests. In particular, it illustrates the interplay between the services composing the demonstrator.

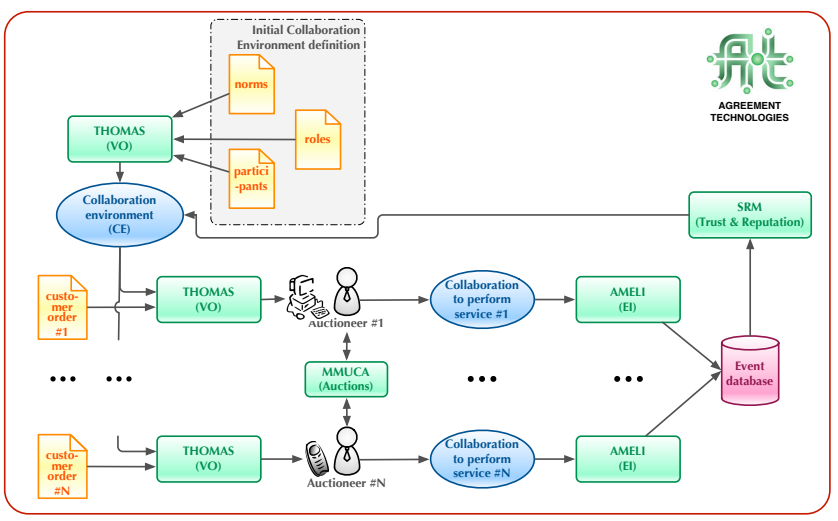

Figure 2. ABC4MAS architecture.

\section{ACKNOWLEDGMENT}

Funded by AT (CONSOLIDER CSD2007-0022), TIN2009-14702-C02-01, TIN2009-13839-C03-01, PROMETEO/2008/051 and 2009-SGR-1434.

\section{REFERENCES}

[1] E. Argente, V. Botti, C. Carrascosa, A. Giret, V. Julian, and M. Rebollo, "An abstract architecture for virtual organizations: The thomas approach," Knowledge and Information Systems, pp. 1-25, 2010, 10.1007/s10115-0100349-1. [Online]. Available: http://dx.doi.org/10.1007/s10115010-0349-1

[2] J. Arcos, M. Esteva, P. Noriega, J. Rodriguez-Aguilar, and C. Sierra, "Environment engineering for multiagent systems," Engineering Applications of Artificial Intelligence, vol. 18, no. 2, pp. 191-204, 2005.

[3] A. Giovannucci, J. Cerquides, U. Endriss, M. Vinyals, J. A. Rodriguez-Aguilar, and B. Rosell, "A mixed multi-unit combinatorial auctions test suite," in AAMAS'2009, 2009, pp. 13891390, demo track.

[4] C. Sierra, J. Debenham, A. Fabregues, and J. Madrenes, "The supplier relationships management tool," http://www.iiia.csic.es/SRM. 